\title{
DEPOSITS OF FLUORESCENT ACID-FAST PRODUCTS IN THE NERVOUS SYSTEM AND SKELETAL MUSCLES OF ADULT RATS WITH CHRONIC VITAMIN-E DEFICIENCY
}

\author{
BY \\ LÁRUS EINARSON \\ From the Department of Anatomy, Aarhus University, and the Institute of Brain Pathology \\ of the State Mental Hospital, Aarhus, Denmark
}

Since 1940 there have appeared several reports on neuromuscular lesions in adult rats in experimental vitamin-E deficiency. It is apparent that side by side with changing concepts of vitamin $E$ and the effects of its deficiency in general, a considerable change in the evaluation of the neuromuscular lesions in particular has taken place.

Since the occurrence of neural lesions in experimental vitamin-E deficiency has been seriously doubted, and the earlier work of Einarson and Ringsted (1938) on this subject has been strongly criticized by some leading authorities on vitamin $E$, I have found it absolutely necessary to reinvestigate the matter, with special reference to the changes of the nerve cells, in particular the cell change I have called "cellular lipodystrophy". Two of my recent works on this subject have already been published (Einarson, 1952a and b), and the present paper is the third in the new series.

Ringsted (1935) reported certain neuromuscular disturbances in adult rats that had been kept on a vitamin-E deficient diet for a long period (months). This observation formed the basis of our comprehensive investigation of the whole neuromuscular syndrome and its pathogenesis, and three years later we published our monograph on the effect of chronic vitamin-E deficiency on the nervous system and the skeletal musculature in adult rats (Einarson and Ringsted, 1938). In the following years this publication was supplemented by some brief papers on the subject (Ringsted and Einarson, 1939; Einarson and Ringsted, 1939 ; Einarson, 1941), and the problem has been further dealt with in some of my later work on the structural changes of nerve cells (Einarson, 1945 ; Einarson and Lorentzen, 1946 ; Einarson, 1949).

A very conspicuous and important feature of the histological changes in vitamin E-deficient adult rats is the regular occurrence of peculiar lipoid products in the nervous system, as originally reported and illustrated by Einarson and Ringsted (1938). These products are insoluble in alcohol, stain reddishorange with fat stains, and give an intense orthochromatic colour (e.g., a pure blue or green) with basic aniline dyes, but they are left completely unstained by gallocyanin-chromalum (Einarson and Ringsted, 1938 ; Einarson, 1941, 1949, 1952). The facts, to be demonstrated in this paper, that the products in question are also fluorescent on ultraviolet irradiation and acid-fast when stained by carbol fuchsin add some additional important characteristics. The products mentioned are regularly absent in normal rats or controls receiving protective doses of vitamin $\mathrm{E}$.

This paper will deal mainly with the structural appearance and distribution of these fluorescent, acid-fast deposits in the nervous system and skeletal muscles of vitamin E-deficient adult rats. However, I find it appropriate first to give a brief account of the clinical symptoms displayed by the rats.

\section{The Clinical Picture}

The clinical picture bears the unmistakable stamp of a neuropathic syndrome including hyperkinesia, tremors, some transient muscular rigidity, increasing hypaesthesia and hypalgesia, prominent ataxia and dysmetria, progressive paralyses and muscular atrophies which, in the final stage, show a marked symmetrical distribution. Finally the animals become exhausted and mentally very slow or sluggish. Also there occurs a symmetrical loss of hair and other trophic disturbances in the skin (atrophy and ulcerations), and finally a lowered circulation in the front and hind paws, the skin becoming cooler than usual and cyanotic. Incontinence of the bladder develops in most of the animals.

In our detailed description of the clinical picture we divided its development into four stages, but it 
will not be further described in this paper. I shall merely add that the whole syndrome is an extremely chronic one. Its onset is insidious after many months on the experimental diet, and it proceeds very slowly; from its first appearance it takes six to 15 months to develop completely. The neuromuscular disturbances mostly begin caudally and progress towards the head, affecting the front and hind legs and hind quarters in a remarkably symmetrical manner. The mentality and general thriving of the animals are not essentially affected until the end of the third or the beginning of the fourth stages. Very small daily doses of vitamin E will prevent the appearance of the disease, but even large doses fail to cure manifest disturbances, and vitamin $\mathrm{E}$ arrests the development of the disturbances only if given very early in the disease.

The whole clinical picture as summarized above deviates rather sharply from the paralysis in the young suckling rats described by Evans and Burr (1928), or the nutritional muscular dystrophy in guinea-pigs and rabbits reported by Goettsch and Pappenheimer (1931); both these are conditions of primary myopathy probably without neural lesions. In the sucklings the paralysis has an acute onset appearing as a sudden calamity towards the end of the lactation period, on about the twentieth to the twenty-fifth day of life, affecting mainly the musculature of the body wall and the posterior extremities in a diffuse manner. Some of the paralyzed sucklings recover spontaneously and completely, others recover with permanent paralyses, and others die without any premonitory symptoms. In the guineapigs and rabbits the disease also has an acute onset and a comparatively rapid course, the result being widely distributed changes of a severe muscular dystrophy, a condition cured by vitamin E. We have repeatedly emphasized these differences from the clinical syndrome in the adult rats. Besides the clinical difference, the deviations of the muscle changes in the suckling rats, the guinea-pigs, and rabbits from the picture of the muscle changes in the adult rats have been clearly emphasized by Demole (1939), Demole and Pfaltz (1940), and Mackenzie, Mackenzie, and McCollum (1940).

Thus, the clinical picture displayed by adult and old rats that have been kept on a vitamin-E deficient diet for a long time is by no means confined to the symptoms and course of a simple or uncomplicated myopathy, although it includes some essential myogenic lesions (phase II). On the contrary it bears the unmistakable stamp of disturbances of the nervous system, and, in my opinion, the completely negative response to vitamin-E therapy clearly demonstrates that early, irreversible neural lesions must be involved in the disease. As a matter of fact my histological examinations have again confirmed this point of view so as to leave no room for doubt.

Before giving an account of the histological changes, in particular the structural appearance of the fluorescent, acid-fast deposits mentioned above, I must preface some remarks on technique.

\section{Technique}

For the demonstration of fluorescent substances the large fluorescence apparatus " lux U.V." of Reichert was used. The source of light is the lux U.V. mercury high pressure burner, and the equipment of light filters consists of the well known Reichert combination, i.e., a chamber with copper sulphate solution as a red blocking filter, two ultra-violet filters (nos. 8079 and 8080), and a matt-white filter (no. 8078) for microscopy with the usual visible light.

The material was fixed in formalin or formalin vapour and either examined as frozen sections mounted in water or glycerine, or it was embedded in paraffin and the sections mounted in non-fluorescent immersion oil (Reichert), or in pure liquid paraffin which does not cause any disturbing fluorescence even after prolonged irradiation. In every case the sections were mounted on Reichert's non-fluorescent slides (U.V.-glass), which the filtered ultra-violet light easily penetrates.

It is noteworthy that before examining the sections in the fluorescence apparatus they may be stained with gallocyanin-chromalum $(p \mathrm{H} \mathrm{1.64)}$ if wanted, since this staining, in spite of the chromalum in the solution, weakens only to a minimum degree the primary fluorescence of the deposits. Of course the sections must be thoroughly washed in water before examination in the fluorescence apparatus. This makes it possible to examine the same nerve cell and compare its structural changes alternately in ultra-violet and visible light (Figs. 3, 6, 7). In thicker sections $(10-20 u)$ the stained Nissl substance may partly conceal the fluorescent deposits, but otherwise the gallocyanin-chromalum staining does not essentially interfere with the fluorescence of these deposits. This circumstance once again demonstrates the high degree of selectivity of the gallocyanin-chromalum staining. By any other of the usually applied staining methods for basophilic cell structures (e.g., the various haematoxylin methods, basic aniline dyes) the primary fluorescence of the products in question is totally lost.

For the demonstration of acid fastness the following procedure was adopted :-(1) Staining of paraffin sections in Ziehl-Neelsen's carbol fuchsin for two hours at $60^{\circ} \mathrm{C}$. followed by washing in water ; (2) decolorization in $3 \%$ hydrochloric acid-alcohol (i.e., $3 \mathrm{ml}$. concentrated $(37 \cdot 5 \%) \mathrm{HCl}$ to $97 \mathrm{ml} .70 \%$ alcohol) for 15 minutes to one hour ; (3) after washing in running tap water for a few minutes the slides are placed in distilled water ; (4) counterstaining with gallocyanin-chromalum

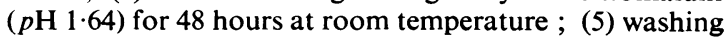


in distilled water, dehydration, clearing, and mounting as usual.

Acid-fast deposits stain an intense red, while nuclear chromatin, nucleoli, and the Nissl substance appear in the usual blue (Figs. A, B, C, D, E). On account of its exquisite progressivity and selectivity the counterstaining with gallocyanin-chromalum, a stoicheiometrical staining reaction for nucleic acids, is superior to any other method for staining basophilic cell structures known to me. It leaves the red staining of the acid-fast products completely unaffected. For the theory and practice of the gallocyanin-chromalum staining, I refer to my recent paper on this subject (Einarson, 1951). My evaluation of the degree of basophilia of the nerve cells (hyperchromasia, chromophoby, chromophily) is exclusively based on this staining method.

\section{The Neuromuscular Lesions}

The picture of the histological changes of the vitamin E-deficient adult rats clearly discloses the fact that the disease is due to a combination of neurogenic and myogenic lesions as originally maintained by Einarson and Ringsted $(1938,1939)$. The muscle changes in particular consist in a late atrophy of spinal origin superimposed on an early muscular dystrophy. The whole complex of neuromuscular lesions in adult rats may be subdivided into the following separate phases according to the site and sequence of their development, although some overlapping in time naturally takes place.

Phase I.-In phase I there is degeneration of the dorsal roots and posterior fasciculi of the spinal cord. As a rule, the process begins in the lumbosacral part of the spinal cord, and gradually progressing towards the head it often involves the cervical segments and even extends into the medulla oblongata. At the lumbosacral levels the entire posterior funiculus is mostly involved, while the lesion, when traced in the direction of the head, gradually becomes more restricted to the fasciculus gracilis, sometimes extending as far as the nucleus gracilis.

Histologically the changes consist of a distinct demyelinization and subsequent fragmentation and reduction of axons. The glial reactions are usually somewhat variable. Mostly, there is a rather marked increase in glial fibres in the demyelinized areas, within which scattered hypertrophic astrocytes are visible. Sometimes the demyelinized areas show a somewhat porous, spongy appearance traversed by the meshwork of glial fibres. As a rule, the histological changes are most marked in the fasciculus gracilis. Numerous scavenger cells, containing abundant normal lipoid breakdown products, staining a brilliant red with scarlet $\mathbf{R}$, are seen, and the peculiar deposits insoluble in alcohol, showing marked affinity for basic aniline dyes and staining reddish-orange with scarlet $R$, are very characteristic. These products are also characterized by their acid fastness when stained by Ziehl-Neelsen's carbol fuchsin according to the procedure described above, and by their bright yellow primary fluorescence on ultra-violet irradiation. These products occur in gliogenous scavenger cells and other phagocytes, in or about astrocytes and oligodendrocytes, and in the walls of the blood vessels within the degenerated areas. They are invariably most abundant and closely packed in the fasciculus gracilis just where the degeneration is most marked (Figs. A and B).

Phase II.--In phase II there is myogenic atrophy of the skeletal muscles, especially pronounced in the hind legs and hind quarters. It begins, as a rule, in the adductors of the thigh, progressively involving more muscles of the posterior extremities as well as the muscles of the hip joint, the lumbar and abdominal regions. Sometimes the anterior extremities are also involved to some extent.

Histologically the muscle changes resemble those of progressive muscular dystrophy. The atrophy is diffuse, with normal, atrophic, and some hypertrophic muscle fibres being irregularly scattered between each other. Migration of nuclei into the muscle fibres is frequently observed, and in longitudinal sections long rows of such nuclei may be seen lying centrally in the muscle fibres. Longitudinal splitting is a constant phenomenon, and hypertrophic muscle fibres may be seen split up into several smaller fibres of varying size. Further, there is a globular fragmentation and disintegration of individual muscle fibres with an increase in hyperchromatic and pyknotic nuclei. Also fibres rich in sarcoplasm, showing signs of vacuolar degeneration, may be seen. Even at this stage of the disease the acid-fast fluorescent products are invariably present in the muscles, first practically confined to the endomysium in which many histiocytes may be filled with them. Later, as they gradually increase in number, they may also be seen lying inside the muscle fibres themselves (Figs. 1 and 2). Sometimes there is an increase of interstitial connective tissue and fat. These dystrophic changes develop rather slowly and mildly compared with the acute, severe, and histologically different muscle changes of the young suckling rats or the guinea-pigs and rabbits.

Thus, dystrophic muscle changes appear at the end of the first or early in the second clinical stage before any changes of the anterior horn cells can be seen. Phase II proceeds by degrees to the next phase (Einarson and Ringsted, 1939). 


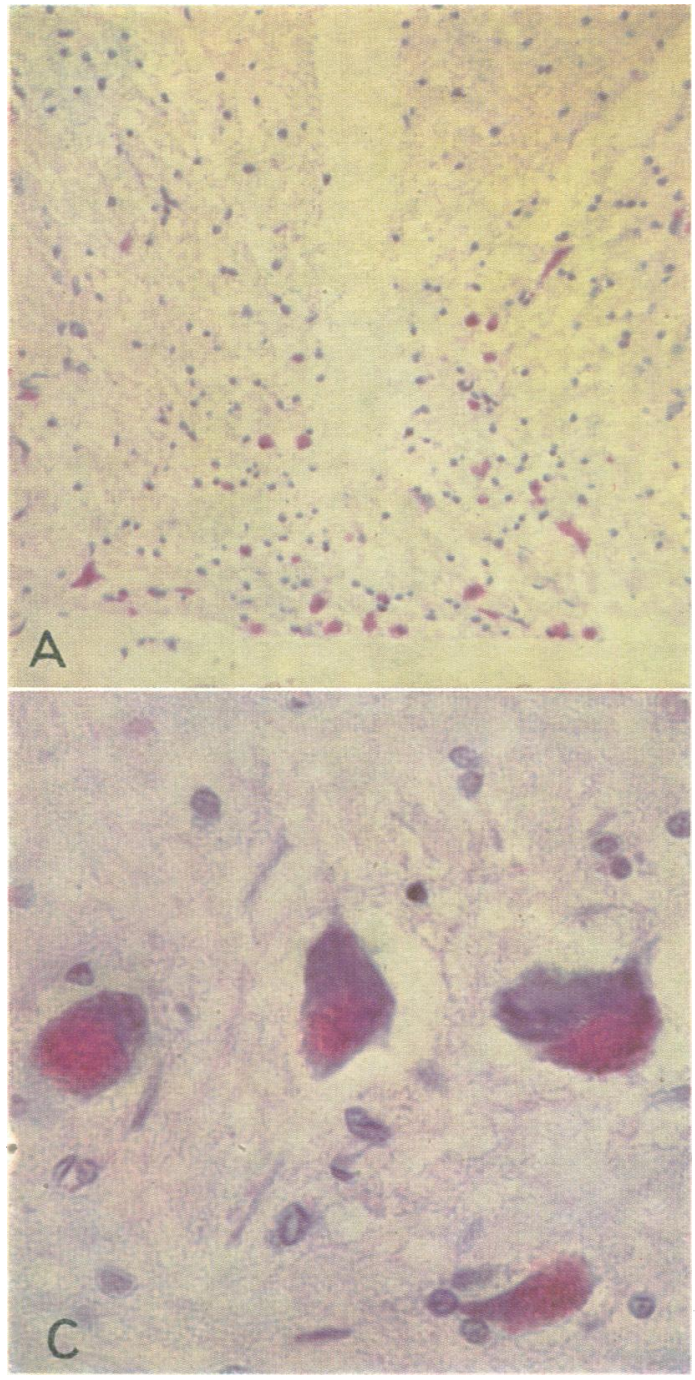

Fig. A.-Acid-fast products in the posterior fasciculi of the spinal cord. Carbol fuchsin and gallocyanin-chromalum $\times 210$.

Fig. B.-Acid-fast products in the fasciculus gracilis. Carbol fucshin and gallocyanin-chromalum $\times 850$.

Fig. C.-Anterior horn cells showing acid-fast substance in the cytoplasm. A considerable amount of basophilic substance is still present. Carbol fuchsin and gallocyanin-chromalum $\times 560$.

Fig. D.-An anterior horn cell distended with acid-fast substance ; its nucleus is shrunken and eccentric, and no basophilic substance (ribonucleic acid) is left in the cytoplasm (cellular lipodystrophy). The acid-fast substance is also seen in some glial cells. Carbol fuchsin and gallocyanin-chromalum $\times 560$.

Fig. E.-Gasserian ganglion showing acid-fast substance in the ganglion cells as well as in the cells of the interstitial connective tissue. Carbol fuchsin and gallocyanin-chromalum $\times 560$.

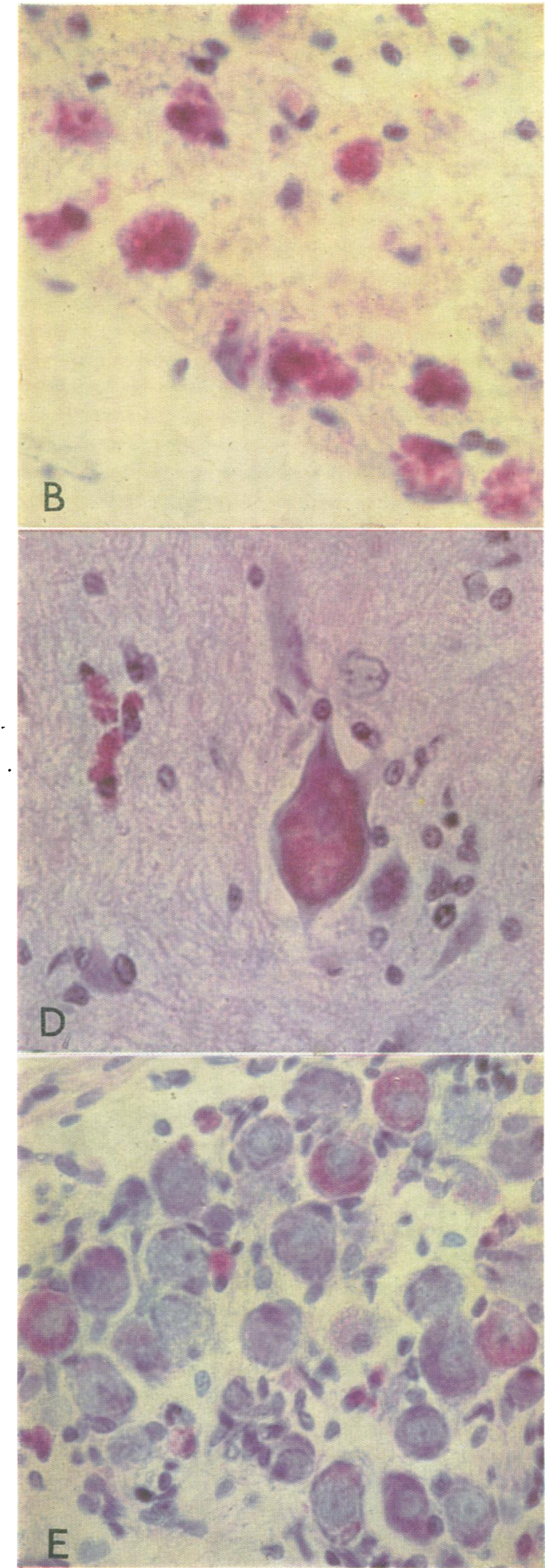

[Facing page 100 


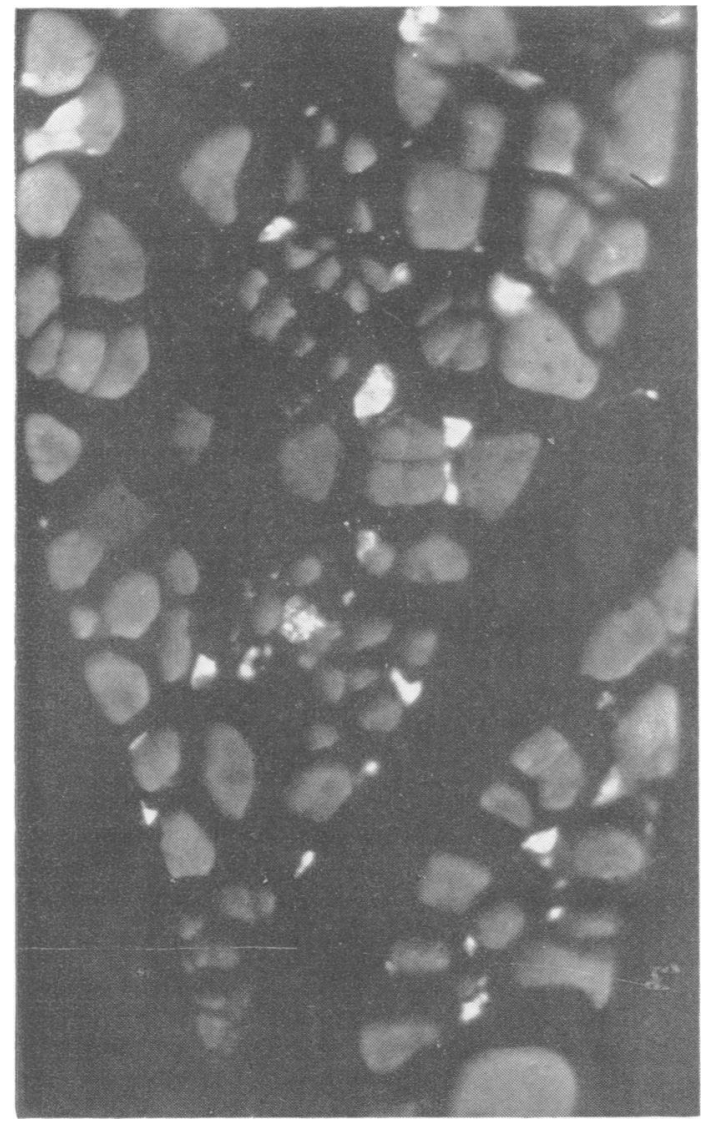

FIG. 1.-Transverse section of the adductor of the thigh showing fluorescent products in muscle fibres and histiocytes of the endomysium and patches of markedly atrophic, less atrophic, and normal muscle fibres. Unstained section. Reichert's "lux U.V." $\times 415$.

Phase III.-In phase III there are slowly developing changes of the visceral and somatic motor and sensory cells of the spinal cord and brain stem. As a rule the cell changes first appear in the lumbar and sacral parts of the cord, and proceeding towards the head they gradually involve the whole spinal cord and brain stem. As the changes progressively involve more parts of the nervous system, and the number of nerve cells affected increases, the changes in each individual cell likewise become more marked. Although the visceral as well as the big motor cells, in particular the anterior horn cells, usually show the most pronounced changes, the cells of the posterior horns, the sensory nuclei of the brain stem, the spinal and cranial ganglion cells are also distinctly affected. It is of special interest to point out the early appearance and marked intensity of the cell changes in the intermediary, visceral columns of the spinal cord (Figs. 3 and 4), a feature particularly emphasized and illustrated by Einarson (1941). Exactly the same applies to the visceral nuclei of the brain stem, e.g., the dorsal motor nucleus of the vagus.

The cell changes referred to are somewhat variable, although they mostly pass through several consecutive structural stages. From the usual condition of normal staining the nerve cells pass through stages of hyperchromasia and chromophoby (reduced basophily) followed by stages of markedly increasing basophily leading to extreme chromophily and cell sclerosis. Gradually they lose their intense staining and end in irreparable cell atrophy with more or less corkscrew-shaped dendrites. At any of these stages the acid-fast fluorescent products may appear in the cytoplasm, often as an early structural sign in otherwise normal-looking cells. From a small cytoplasmic deposit the products gradually increase until the majority of the cells, while becoming chromophilic and atrophic, finally become more or less filled with them, while there is a simultaneous disappearance of the Nissl substance. This cytoplasmic change is accompanied by eccentric displacement, hyperchromatosis, and shrinkage of the nucleus. Thus, the nucleic acids of the cytoplasm are gradually replaced by the acidfast fluorescent products, many cells showing mere

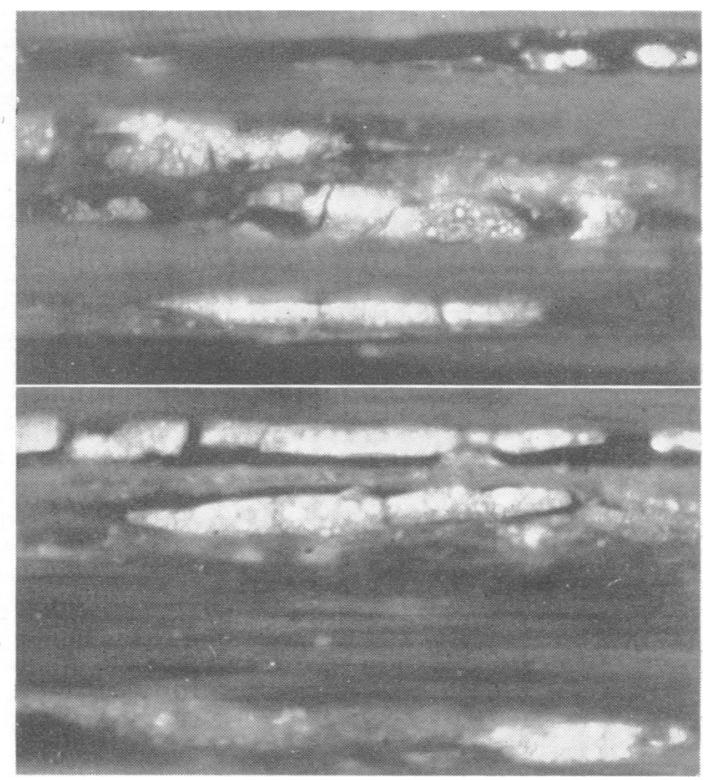

FIG. 2.-Longitudinal section of the adductor of the thigh showing fluorescent products lying inside the muscle fibres as well as in the endomysium. Unstained section. Reichert's "lux U.V." $\times 415$. 


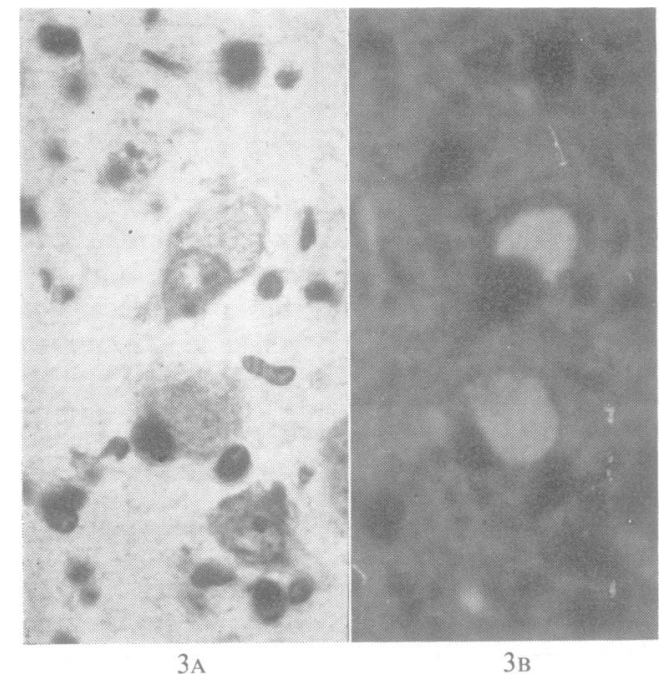

FIG. 3.-Nerve cells from the intermedio-medial cell groups (autonomic zone) of the spinal cord. The cytoplasm is ristended with lipoprotein substance; the nuclei show an eccentric displacement. (A) In daylight the lipoprotein is unstained. (B) In ultra-violet light the same lipoprotein is strongly fluorescent. Gallocyanin-chromalum and "lux U.V." $\times 625$.

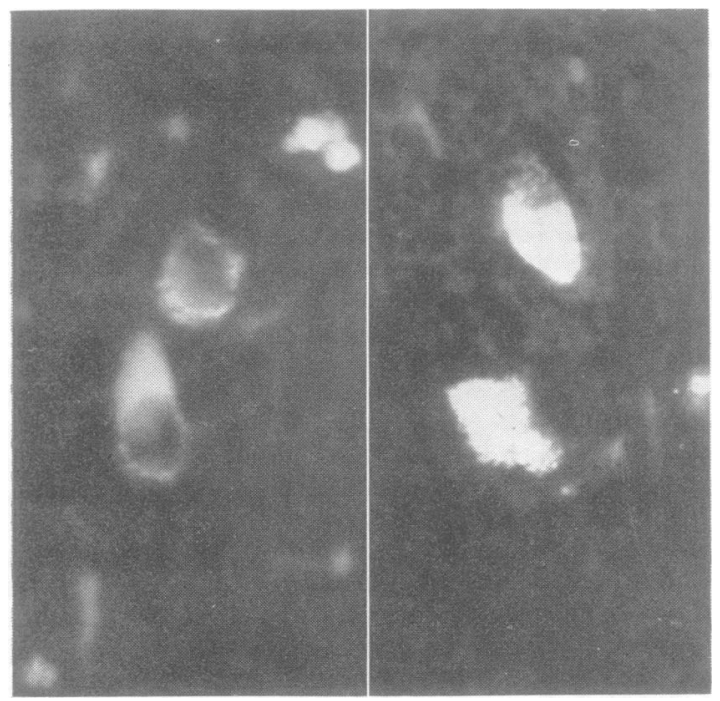

FIG. 4.-Nerve cells from the intermedio-lateral column of the spinal cord. The cytoplasm is filled with fluorescent products; the nuclei are more or less eccentric. Unstained section. "Lux U.V." $\times 625$.

remnants of basophilic substance round the hyperchromatic, shrunken nucleus, or none at all. As far back as 1938 it was clearly pointed out by Einarson and Ringsted that this lipoid degeneration might completely dominate the stages of extreme chromophily and cell sclerosis. We have called this cell change " cellular lipodystrophy" (Einarson and Lorentzen, 1946 ; Einarson, 1949).

The acid-fast fluorescent products appear as diffuse or densely packed deposits of fine and coarse granules or globular particles in the cytoplasm of the nerve cells in or about astrocytes, oligodendrocytes, microglia and histiocytes, and in the walls of the blood vessels (Figs. 3-7, C-E). Unstained they show a greyish-yellow, sometimes rather dark colour. Besides their acid-fastness when stained by Ziehl-Neelsen's carbol-fuchsin, and their bright yellow primary fluorescence on ultra-violet irradiation, they are characterized by their relative insolubility in lipoid solvents, by staining reddishorange with fat stains, by their affinity for basic aniline dyes, and by the fact that gallocyaninchromalum leaves them completely unstained. These properties strongly suggest that we are dealing with a lipoprotein combination (ceroid) which does not contain nucleic acids (Einarson, 1949, 1952).

The irreparable lipodystrophic changes of the anterior horn cells will unavoidably put their stamp on the picture of the muscular atrophy : in other words, features of a late spinal atrophy are gradually superimposed on the early muscular dystrophy. Thus, the final picture of the muscular atrophy in the vitamin E-deficient adult rats reveals a combination of myogenic and neurogenic changes. The spinal-atrophic features manifest themselves by the occurrence of delimited patches of atrophic

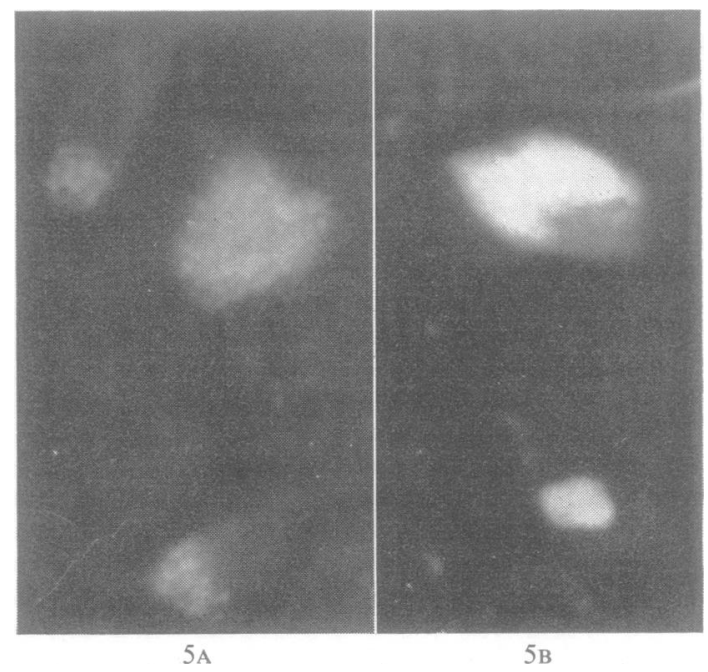

FIG. 5.-Anterior horn cells. Unstained section. "Lux U.V." $\times 625$. (A) With coarsely globular, fluorescent particles in the cytoplasm. (B) With particles coalescing to a compact mass of fluorescent substance ; the nucleus is shrunken and eccentric (cellular lipodystrophy). 


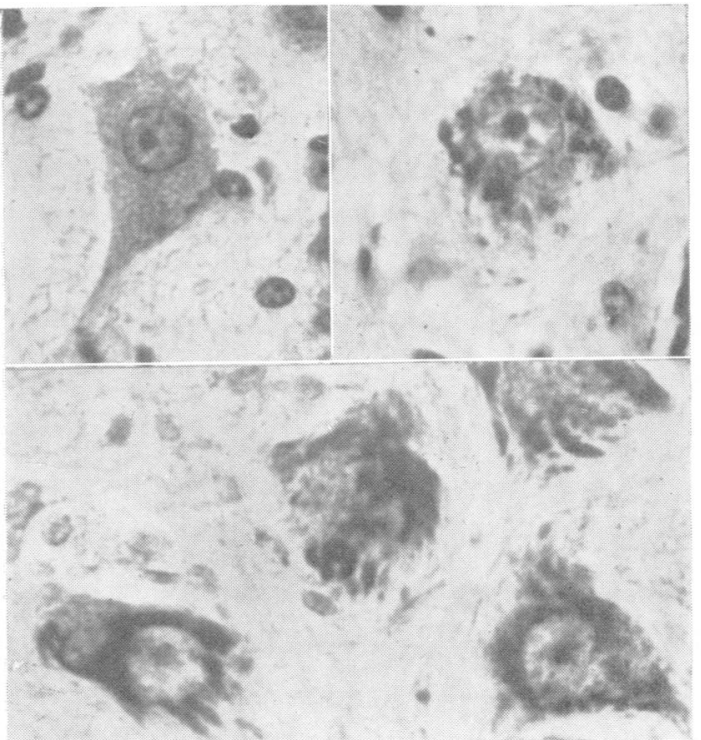

$6 \mathrm{~A}$
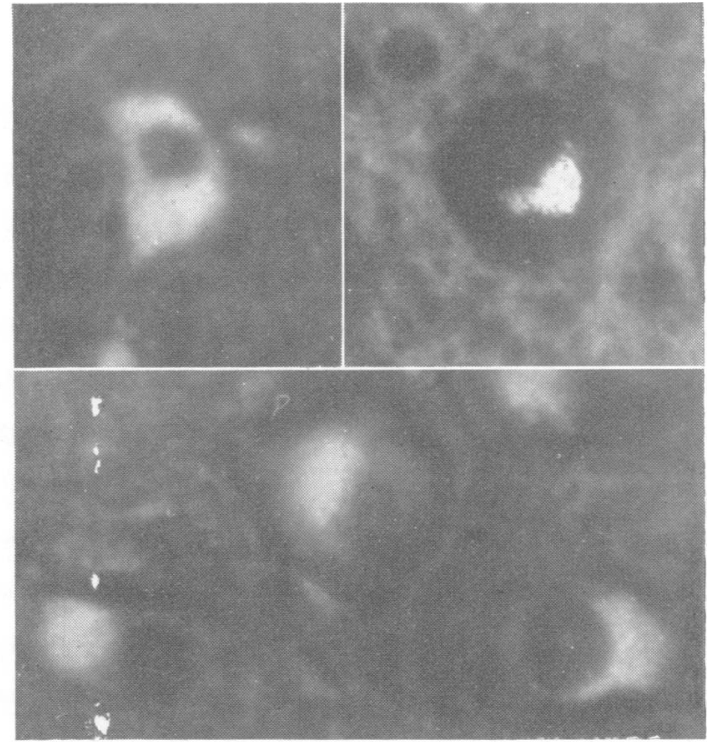

6B

Fig. 6.-Anterior horn cells with more or less lipoprotein substance in the cytoplasm. In some of the cells a considerable amount of stainable substance (ribonucleic acid) is still present. (A) In daylight the lipoprotein is unstained. (B) In ultra-violet light the lipoprotein is strongly fluorescent. Gallocyanin-chromalum and "lux U.V." $\times 625$.

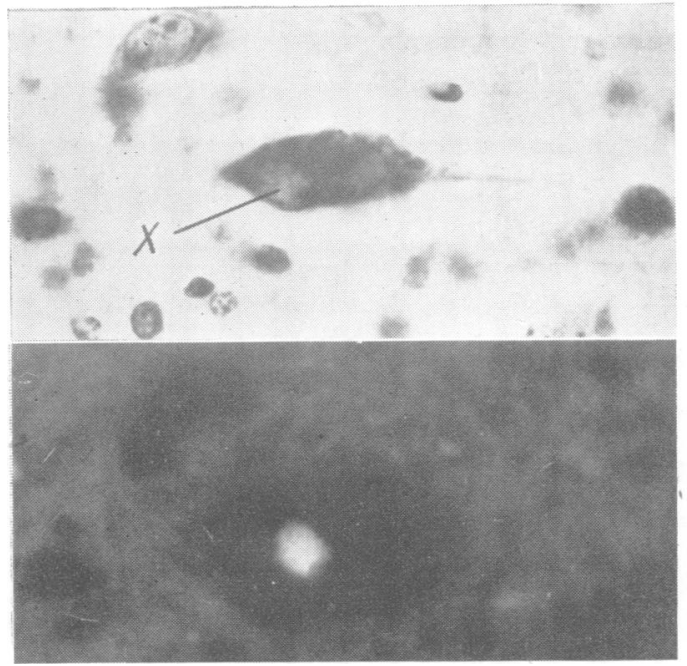

$7 \mathbf{A}$

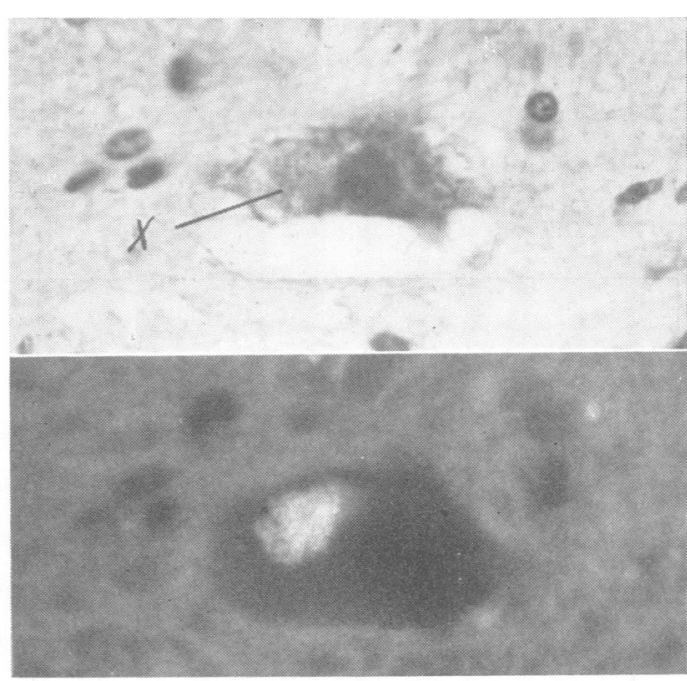

7B

FIF. 7.- Two anterior horn cells in daylight and ultra-violet light respectively with small patches of fluorescent substance ( $x$ ) in the cytoplasm. Gallocyanin-chromalum and "lux U.V." $\times 625$.

(A) The cell is proceeding towards extreme chromophily; the nucleus is hyperchromatic. (B) The cell is proceeding towards irreparable atrophy with a loss of cytoplasmic basophily and hyperchromatosis of the nucleus.

muscle fibres. In the same field may be seen both the less atrophic and markedly atrophic fibres. In the final stage of the disease almost every muscle fibre has become atrophic. Further, the generally enormous increase of large hypolemmal nuclei, with well defined, markedly enlarged, and darkly staining nucleoli is a very characteristic sign that occurs specially in neurogenic atrophy. Moreover, the increase in sarcoplasm, the interruption of the muscle fibres by masses of sarcoplasm surrounding large nuclei with sharply defined, enlarged nucleoli, and numerous fluorescent inclusions, the preserva- 
tion of the striation even in markedly atrophic muscle fibres, as well as the absence of hyaline or waxy degeneration, are features that speak decidedly for the interpretation of the late atrophy as being neurogenic. All these features constitute the spinal, atrophic phase of the process. The fact that it is possible to ascertain the appearance of the late neurogenic features is due only to the circumstance that they are not masked by the relatively mild development of the early myogenic changes.

Phase IV.- In phase IV there is swelling and fragmentation of myelin sheaths in the sciatic nerves, with deposits of fluorescent acid-fast lipoproteins in swollen Schwann cells, and particularly in histiocytes of the endoneurium (Fig. 8). The changes appear late and are comparatively mild, although numerous histiocytes may be seen to be filled with fluorescent particles; these products may also be seen accumulating round blood vessels of the endoneurium, as already shown by Einarson and Ringsted (1938).

Phase V.- In phase V there is a gradual development of severe nerve cell changes in the entire cerebrum. Their distribution is mostly diffuse and irregular. In the thalamus, hypothalamus, caudate and lenticular nuclei, as well as in the rhinencephalon and the non-olfactory cerebral cortex (neopallium) of the rats, numerous markedly changed nerve cells may be seen irregularly distributed among still normal or slightly changed cells. However, in the

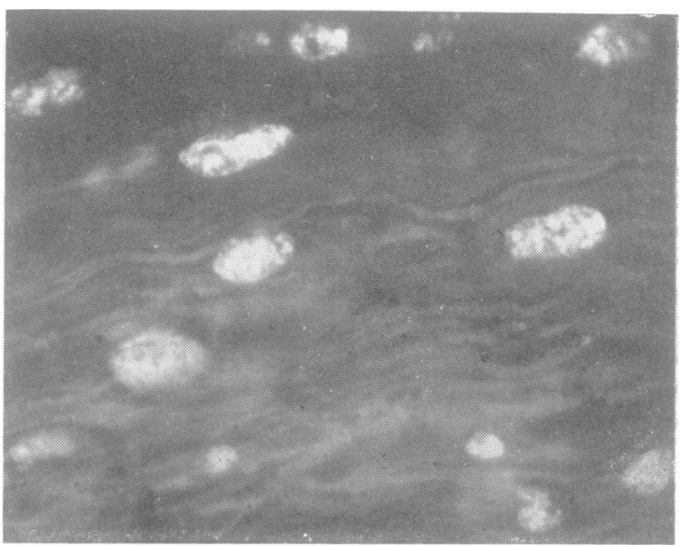

FiG. 8.-Sciatic nerve : many histiocytes of the endoneurium are filled with a strongly fluorescent substance. Unstained section.

“Lux U.V." $\times 625$.

rhinencephalon as well as in the layers of pyramidal cells of the neopallium long horizontal rows of distinctly affected cells may often be seen (Figs. 9 and 10).

Histologically the cell changes are the same aso $\vec{\sigma}$ met with in the spinal cord and brain stem, and further description is not necessary. I may jus $\varnothing$ mention that the number of extremely chromophilio 옹 cells is decidedly increased as compared with the normal, and the fluorescent acid-fast products $\vec{z}$ appear in the cytoplasm of the nerve cells in almose every region of the cerebrum. They are also to be found in or about the main types of glial cells, ans

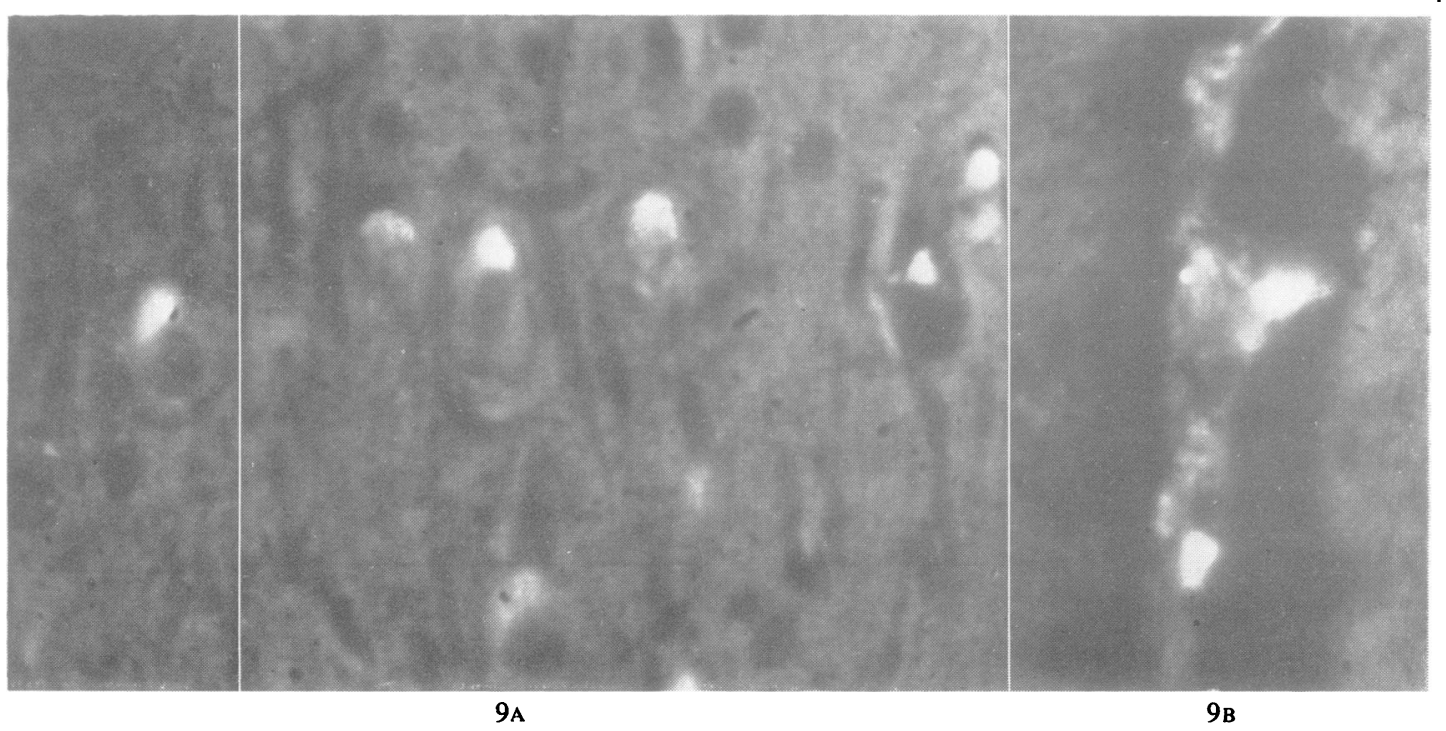

Fig. 9.-Neopallium. (A) Pyramidal cells showing more or less of strongly fluorescent substance in the cytoplasm. (B) Cortical blood vessel surrounded by fluorescent products. Unstained section. "Lux U.V." $\times 625$. 


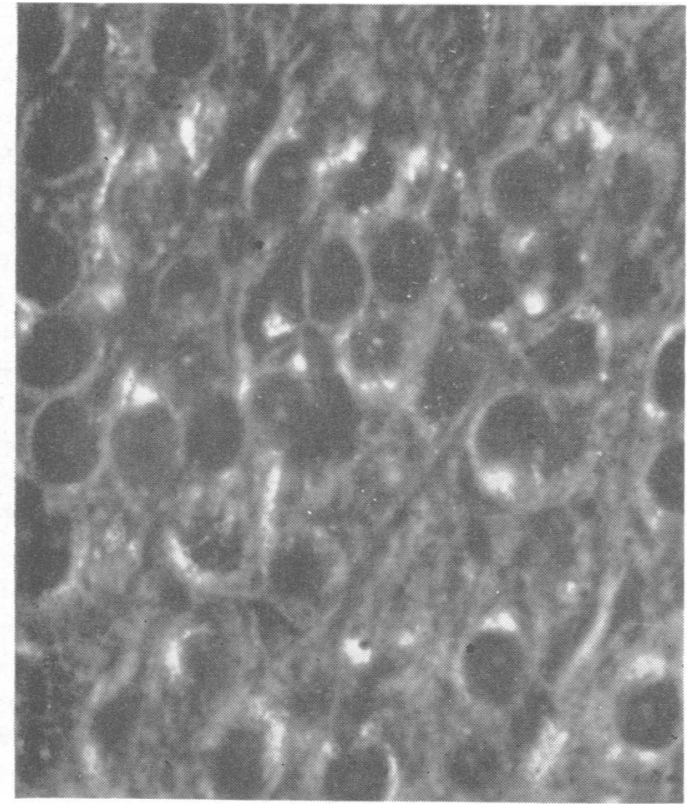

FIG. 10.--Rhinencephalon: nerve cells of the cortex showing fine granules of fluorescent substance diffusely distributed in the cytoplasm, especially round the nuclei. Small fluorescent particles are also lying in the glial cells. Unstained section. "Lux U.V." × 625 .

in or round the walls of the blood vessels. Although numerous nerve cells containing considerable amounts of the fluorescent lipoproteins may be observed almost everywhere, the intracellular quantities of the deposits in question are on the whole somewhat less than in the spinal cord and brain stem. However, some nerve cells completely filled with these products may always be seen, and besides the more severely changed cells a great number contain a trace of the fluorescent acid-fast substance. Thus, "cellular lipodystrophy" is a widely distributed cell change in the cerebrum of the vitamin E-deficient adult rats.

Phase VI.-The late appearance of cell changes in the cerebellum is not distinctly visible until the final stage of the disease. Although the number of strongly chromophilic Purkinje cells seems to be increased compared with the normal, it is the occurrence of the lipoprotein inclusions that constitutes the most conspicuous feature of the changes. It is remarkable how the fluorescent acid-fast products finally appear in the cytoplasm of such decidedly "lipophobic" nerve cells as the Purkinje cells (Fig. 11). Also, they may be observed in the granular layer and in or about glial cells of the molecular layer, as well as round the blood vessels and in the central nuclei.

In the Purkinje cells the fluorescent acid-fast products are especially situated at the place of origin of the main apical dendrite from the cell body, often reaching down to the nuclear membrane and replacing more or less the basophilic nuclear cap. Sometimes they occupy a considerable portion of the cytoplasm of a Purkinje cell, even surrounding the whole nucleus. However, in each individual Purkinje cell we are mostly dealing with a comparatively small amount of fluorescent acid-fast substance, and, in consideration of the decidedly "lipophobic" character of this particular cell type, this is really to be expected. But, considering the fact that the most of the Purkinje cells show
Fici. 11.-Cerebellum. (A) Section perpendicular to the surface of a folium. Purkinje cells showing fluorescent substance especially situated at the place of origin of the main dendrite from the cell body. (B) Section parallel to the surface of a folium. Purkinje cells with considerable amounts of perinuclear fluorescent substance. Unstained sections. "Lux U.V." $\times 625$.

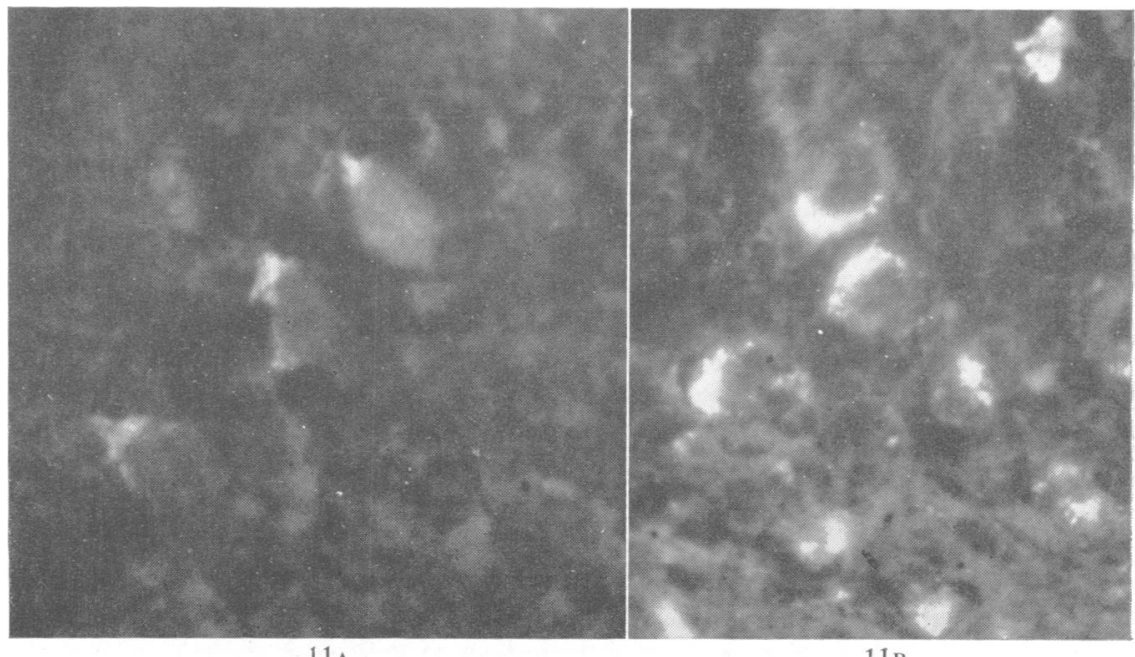


these characteristic deposits, the phenomenon must be said to be extensively distributed in the cerebellar cortex.

\section{Discussion}

The results originally reported by Einarson and Ringsted $(1938,1939)$ were essentially confirmed by Monnier $(1940,1941,1942)$ and by de GutiérrezMahoney (1941), who found degeneration of the posterior fasciculi of the spinal cord, sclerosis, and irreparable atrophy of the nerve cells of the anterior horns and intermedio-lateral columns and slight demyelinization of the dorsal roots and peripheral nerves, but they did not mention the occurrence of any lipoid products in the nervous system of the rats. Monnier agrees with us that the muscular atrophy is a combination of myogenic and neurogenic changes.

Soon, however, the correctness of our results and concepts was seriously doubted by Mason (1942), and completely denied by Wolf and Pappenheimer (1942) and by Pappenheimer (1943). The last mentioned authors stated with certainty that, under the experimental conditions obtaining in their laboratory, lesions of the central nervous system did not occur in vitamin E-deficient rats at any age, and they actually regarded our findings as simply due to technical artefacts and misinterpretations. The results and conclusions of Wolf and Pappenheimer were supported by Harvey and Perryman (1944), and Lecoq and Isidor (1949), and recently the results and considerations of Einarson and Ringsted (1938) have been seriously criticized by Ruppel (1949) and Markees (1950), who apparently adhere to the interpretations and criticism of Wolf and Pappenheimer and Mason.

I shall not discuss these critical views in the present paper, but merely refer to my recent review of the concepts of the neuromuscular lesions in experimental vitamin-E deficiency (Einarson, 1952), in which I have clearly shown how unwarranted this criticism of Einarson and Ringsted's early work was and that the original interpretations of Einarson and Ringsted were essentially correct. Concerning the histological aspect of the effect of vitamin-E deficiency on the nervous system, the studies criticizing Einarson and Ringsted's results have actually failed, as the authors either have overlooked the occurrence of the neural lesions or they have misunderstood our interpretations. Moreover, it appears to me that some of the authors (e.g., Lecoq and Isidor) have not made any serious attempt to reproduce our experimental conditions. I also think that unfamiliarity with, and misunderstanding of, the gallocyanin-chromalum staining, which in an essential way deviates from the tech- nique usually applied by the majority of investigators, invalidated much of the criticism.

Some essentials of our earlier observations have now been confirmed by the leading authors among those who formerly used to regard our findings as a mistake. Thus, Wolf and Pappenheimer (1945) and Pappenheimer and Victor (1946) have ascertained the regular occurrence of considerable amounts of acid-fast substance in the motor cells of the spinal cord and medulla oblongata in adult rats maintained for long periods on a vitamin-E deficient diet, and its absence in controls receiving protective doses of wheat germ oil or tocopherol. Indeed, the American authors did not find these products until they used carbol fuchsin for staining followed by decolorization in acid-alcohol. The fact that Wolf and Pappenheimer completely overlooked the occurrence of the lipoprotein inclusions in their first paper is partly due, I think, to their staining technique. They used toluidin blue and cresyl violet for the nerve cells, and these basic dyes stain the Nissl substance and the acid-fast products in approximately the same way, i.e., in a pure orthochromatic blue. Hence it may be very difficult or practically impossible to distinguish between the acid-fast products and true Nissl substance in such preparations. Gallocyanin-chromalum, on the other hand, leaves the acid-fast products completely unstained, and hence we could easily distinguish them from the selectively stained Nissl substance. We have repeatedly emphasized this very important fact, and the unstained, lipoprotein inclusions of the nerve cells were illustrated in our monograph. We also illustrated them in the sciatic nerves after staining with scarlet R. (Einarson and Ringsted, 1938). But all this was completely overlooked by Wolf and Pappenheimer (1942), as well as by all the other authors quoted above. Moreover, in their later papers Pappenheimer and his associates (1945, 1946) have completely omitted to consider our earlier findings.

I wish to emphasize that the fluorescent acid-fast products are much more widely distributed in the nervous system of the vitamin E-deficient rats than maintained by Wolf and Pappenheimer and Pappenheimer and Victor, who found them only in the motor cells of the spinal cord and medulla oblongata. In this paper I have demonstrated that the products in question are distributed practically all over the nervous system (Phases III-VI).

Furthermore, the degeneration of the posterior spinal fasciculi and dorsal roots (phase I), as originally reported by Einarson and Ringsted (1938), has been fully confirmed by Luttrell and Mason (1949) and by Malamud, Nelson, and 
Evans (1949). Apart from the fact that the authors quoted do not have a word to say about the occurrence of any fluorescent acid-fast products in the posterior fasciculi, I have nothing to remark; as for the rest, their findings are in complete agreement with our earlier observations. But the vast distribution of the fluorescent acid-fast products in nerve cells, glial cells, histiocytes, and the walls of the blood vessels practically all over the nervous system (phase III-VI) has apparently escaped their attention, although Luttrell and Mason have used Kinyoun's carbol fuchsin staining, and Malamud and others mention a slight increase in fat content of the anterior horn cells. These authors maintain, just as Pappenheimer and his associates (1945, 1946) do, that there are no changes whatever to be found in the brain in experimental vitamin-E deficiency.

A very striking characteristic of the neural changes produced by experimental vitamin-E deficiency is their early irreversibility. Serious clinical disturbances develop at a time at which structural changes are barely or not at all detectable by our present technique, and yet the disease progresses in spite of the therapeutic administration of vitamin E. In my opinion, this demonstrates that the changes must start at a submicroscopic, biochemical level, and their structural manifestation at the microscopic level is essentially a function of the time factor. Whether the electron-microscope might reveal some subtle, initial changes I am not able to say.

This early irreversibility of the experimental changes must be borne in mind as regards the clinical treatment of patients with vitamin $E$. Whether a deficiency or a defective utilization of vitamin $\mathrm{E}$ (dysvitaminosis) is a concurrent factor in the development of neural or neuromuscular disorders in man is still questionable. But even if this actually were so we could not expect much from vitamin-E treatment alone, since the lesions produced in adult rats, by deprivation of the vitamin itself, are practically irreversible and uninfluenced by the therapeutic administration of vitamin E. This was clearly emphasized by Einarson and Ringsted (1938), but it has apparently been overlooked by the majority of those clinicians who have been engaged in treating patients suffering from neuromuscular disorders with vitamin $E$. The largely negative or equivocal results from the clinical treatment with vitamin $\mathrm{E}$ are completely in accordance with the therapeutic experiments on rats, in which the disease is incurable by vitamin $E$.

Furthermore, I wish, in particular, to emphasize the following points:-(1) The almost ubiquitous occurrence of fluorescent acid-fast substance (lipoprotein) in the nervous system of adult rats that have long been kept on a vitamin-E deficient diet, and its regular absence in controls receiving protective doses of vitamin E. (2) In the cytoplasm of the nerve cells the amount of fluorescent acid-fast substance is inversely proportional to that of the nucleic acid; in other words as the fluorescent acid-fast substance increases, the ribonucleic acid of the cytoplasm gradually disappears, a cell change accompanied by a more or less eccentric displacement, hyperchromatosis, and shrinkage of the nucleus ("cellular lipodystrophy"). (3) The early appearance and marked intensity of the cell changes in the autonomic centres of the spinal cord and brain stem: thus, in the entire intermediolateral cell columns, as well as in the medial cell groups of the intermediary zones of the spinal cord, the cells appear completely filled with fluorescent acid-fast substance, already at the end of the second or the beginning of the third clinical stages.

The last point (3) might be of great importance in the whole syndrome of the vitamin-E deficient rats, a possibility duly considered by Einarson and Ringsted (1938), Evans (1940), and Einarson (1941), who supposed that a primary injury to the autonomic nervous system might be involved in this particular deficiency. It might be surmised that a disturbance of the balance of autonomic regulations concerned with the vasomotor and metabolic controls of the tissues took place. Actually, the work of Martin (1946) on the action of prostigmine in the nutritional muscular dystrophy of vitaminE deficiency is in support of this hypothesis. Also, I refer to my recent considerations on this particular point (Einarson 1949, 1952).

In conclusion, I think it is justifiable to state that vitamin $E$ is essential to the normal health and the structural and functional integrity of the nervous system in the rat. This cannot be a surprise since vitamin $\mathrm{E}$ is involved in certain fundamental biological oxidations, and possibly in some phosphorylations as well, although its ultimate physiological action is still obscure. The fact that experimental vitamin-E deficiency produces conspicuous microscopic lesions in many organs and tissues clearly shows that its function in tissue metabolism must be of an almost universal importance in the organism. The idea that the nervous system should escape the injurious effect of experimental vitamin-E deficiency is, in my opinion, incompatible with our present knowledge of the physiology and biochemistry of vitamin $\mathrm{E}$ (Einarson, 1952).

Finally the fluorescent acid-fast products des- 
cribed in this paper are not to be regarded as something specific for vitamin-E deficiency. Although the products deviate from the usual soluble lipochrome, which stains a brilliant red with scarlet R, they may be observed in human necropsy material, both in the nervous system and elsewhere, more or less mixed with the usual lipochrome substance. Possibly, the commonly occurring lipochrome is a mixture of substances, some of which are fluorescent and acid-fast and relatively insoluble in the usual fat solvents. But, in order to produce such fluorescent and acid-fast products in large amounts in the tissues of animals, experimental chronic vitamin-E deficiency is a most effective and important procedure.

\section{Summary}

Neuromuscular lesions, in particular the regular occurrence of deposits of fluorescent, acid-fast products in the nervous system and skeletal muscles of adult rats that have long been kept on a vitamin$\mathrm{E}$ deficient diet, are reported. The findings generally confirm the earlier observation and description by Einarson and Ringsted (1938) of these neuromuscular lesions, and in particular of lipoid products in the nervous system of adult rats in chronic vitamin-E deficiency. Furthermore, it is demonstrated that the nerve cell changes and the acid-fast deposits are far more widely distributed in the nervous system than was previously thought.

A brief account is given of the clinical symptoms displayed by rats, and some remarks on technique.

The histological examination clearly shows that the disease is due to a combination of neurogenic and myogenic lesions as originally maintained by Einarson and Ringsted (1938, 1939). The lesions consist of degeneration of the dorsal roots and posterior fasciculi of the spinal cord, myogenic changes of the skeletal muscles followed by pronounced changes of the visceral and somatic motor and sensory cells of the spinal cord and brain stem, as well as of the cells of the spinal and cranial ganglia, and gradually features of neurogenic muscular atrophy are superimposed on the earlier myogenic changes. Somewhat later there appear mild changes in the sciatic nerves and marked diffusely distributed cell changes in the central nuclei of the brain and the entire cerebral cortex, followed by mild cell changes in the cerebellum.

Everywhere the changes are highly characteristic of the lipoid products. Besides staining reddishorange with fat stains they are characterized by their relative insolubility in lipoid solvents, their affinity for basic aniline dyes (orthochromatic staining), their acid-fastness when stained by ZiehlNeelsen's carbol fuchsin, their bright, yellow, primary fluorescence on ultra-violet irradiation, and by the fact that they are left completely unstained by gallocyanin-chromalum, a stoicheiometrical staining reaction for nucleic acids. These properties strongly suggest that we are dealing with a lipoprotein combination (ceroid) which does not contain nucleic acids.

The fluorescent acid-fast products appear as fine, rounded granules or coarsely globular particles, sometimes coalescing to compact masses. They lie in the cytoplasm of the nerve cells, in or about glial cells and histiocytes, and in the walls of the blood vessels, and they occur almost all over the nervous system. In the muscles they lie both inside and outside the muscle fibres. They are regularly absent in controls receiving protective doses of vitamin $\mathrm{E}$.

In the nerve cells the progressive increase of the fluorescent acid-fast substance invariably involves a corresponding decrease in the ribonucleic acid of the cytoplasm until it completely disappears, a cell change accompanied by more or less eccentric displacement, hyperchromatosis, and shrinkage of the nucleus ("cellular lipodystrophy". The early appearance and marked intensity of this cell change in the autonomic centres of the spinal cord and brain stem is particularly emphasized.

The changes must start at a submicroscopic, biochemical level, and their early irreversibility is pointed out.

Fluorescent, acid-fast products comparable with those described in rats may be observed in human necropsy material, more or less mixed with the commonly occurring lipochrome.

\section{REFERENCES}

Demole, V. (1939). C. R. 3e Congr. neurol. int., Copenhague, p. 927. Munksgaard, Copenhagen.

—_, and Pfaltz, H. (1940). Rev. méd. Suisse rom., 60, 464.

Einarson, L. (1941). Nord. Med., 11, 2082.

- (1945). Acta Jutlandica, 17, no. 1, pp. 108-125.

- (1949). Acta orthop. scand., 19, 55.

-(1951). Acta path. microbiol. scand., 28, 82.

- (1952a). Acta psychiat. Kbh., Suppl. No. 78.

- (1952b). Ugeskr. Laeg., 114, 1186.

_-, and Lorentzen, K. A. (1946). Acta Jutlandica, 18, no. 4, pp. 62-66.

- - and Ringsted, A. (1938). Effect of Chronic Vitamin E Deficiency on the Nervous System and the Skeletal Musculature in Adult Rats. Levin and Munksgaard, Copenhagen.

- - (1939). C. R. $3^{\mathrm{e}}$ Congr. neurol. int., Copenhague, p. 937.

Evans, H. M. (1940). J. Mt Sinai Hosp., 6, 233.

-_, and Burr, G. O. (1928). J. biol. Chem., 76, 273.

Goettsch, M., and Pappenheimer, A. M. (1931). J. exp. Med., $54,145$.

Gutiérrez-Mahoney, W. de (1941). Sth. med. J. Bgham, Ala.,

34, 389. 99, 631 . 
Lecoq, R., and Isidor, P. (1949). Ann. N.Y. Acad. Sci., 52, 139.

Luttrell, C. N., and Mason, K. E. (1949). Ibid., 52, 113.

Mackenzie, C. G., Mackenzie, J. B., and McCollum, E. V. (1940). Proc. Soc. exp. Biol. N.Y., 44, 95.

Malamud, N., Nelson, M. M., and Evans, H. M. (1949). Ann. N.Y. Acad. Sci., $52,135$.

Markees, S. (1950). Int. Z. Vitaminforsch., 22, 335.

Martin, G. J. (1946). Exp. Med. Surg., 4, 326.

Mason, K. E. (1942). Yale J. Biol. Med., 14, 605

Monnier, M. (1940). Arch. Sci. phys., nat., 22, 252.
Monnier, M. (1941). Z. Vitaminforsch., 11, 235.

(1942). Schweiz. Arch. Neurol. Psychiat., 49, 284.

Pappenheimer, A. M. (1943). Physiol. Rev., 23, 37.

- and Victor, J. (1946). Amer. J. Pathol., 22, 395.

Ringsted, A. (1935). Biochem. J., 29, 788.

and Einarson, L. (1939). C. R. 3e Congr. neurol. internat., Copenhague, p. 932.

Ruppel, W. (1949). Arch. exp. Path. Pharmak., 206, 584.

Wolf, A., and Pappenheimer, A. M. (1942). Arch. Neurol. Psychiat., Chicago, 48, 538.

- - (1945). J. Neuropath., 4, 402.

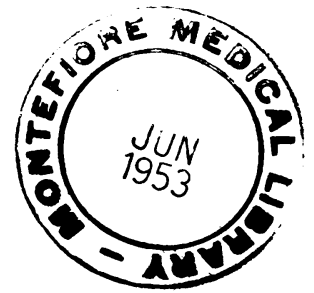

